\title{
Metagenomic-Guided Antibiotics Discovery
}

\section{Zhi-Qiang Xiong*}

Key Laboratory of Synthetic Biology, Institute of Plant Physiology and Ecology, Shanghai Institutes for Biological Sciences, Chinese Academy of Sciences, Shanghai, China

The extensive emergence of multi-drug-resistance pathogens e.g., New Delhi metallo-beta-lactamase 1 (NMD-1) Gram-negative bacteria cause a huge threat to public health. One direct course of action to treat drug-resistant pathogenic infections and avoid an epidemic is to discover new antibiotics [1]. However, during the past 50 years of intensive screening, the pace of antibiotic discovery and development with unique scaffolds is dramatically declining. Since the alreadydiscovered antibiotics are still high-frequency isolated and rapid dereplication techniques for antibiotics are still lacking, the major pharmaceutical companies have noticeably downsized their investment in antibiotic discovery, even stopped doing research in this area [2]. Accordingly, to meet the urgent demand for novel drug, a recent trend in antibiotic discovery from natural sources emphasizes the investigation of metagenomics.

Metagenomics enables direct access to the whole genome of the natural environment through the introduction of environmental DNA (eDNA) into a suitable host and screening of these large eDNA libraries [3]. Discovery of novel natural products (e.g., terragines, violacein, indirubin and turbomycins) with various bioactivities by metagenomic has proven that this method is a good tool for antibiotic discovery [4]. To date, function driven analysis (e.g., bioactivity assay) and sequence driven analysis (e.g., DNA probe screening) are two main strategies for eDNA library screening $[3,5,6]$. Sequence driven analysis could identify the large discrete DNA fragments for the assembly of the intact clusters [7]. Kim et al. [8] reported a general framework for the recovery of large functional DNA fragments from cosmid clones and efficient reassembly of these large fragments using transformation-associated recombination (TAR) in Saccharomyces cerevisiae according to sequence driven analysis. Feng et al. [9] used TAR method to reassemble two characterized eDNA clones (named AB649 and AB185) into a complete PKS cluster and heterologously expressed in Streptomyces albus. Three novel antibiotics fluostatins F, G and $\mathrm{H}$ with antibacterial activities were detected in the assembled clone, but not found in AB649 cultures alone [9].

The development of diverse model systems for eDNA libraries expression could also expand the repertoire of antibiotics. Despite much attempts on eDNA expression in E. coli, Craig et al. [10] explored $\beta$-Proteobacterium Ralstonia metallidurans as a new model system for eDNA expression. Novel antibiotics such as patellamide and pederin were isolated by this new system [10]. Interestingly, cosmids conferred the production of novel antibiotics in $R$. metallidurans were not functioning in E. coli. Although the search for antibiotics by metagenomic methods has only just begun, it is no doubt that metagenomics is a productive source of novel secondary metabolites and has great potential to increase the number of antibiotics in clinical trials.

\section{References}

1. Xiong ZQ, Zhang ZP, Li JH, Wei SJ, Tu GQ (2012) Characterization of Streptomyces padanus JAU4234, a producer of actinomycin X, fungichromin and a new polyene macrolide antibiotic. Appl Environ Microbiol 78: 589-592.

2. Coates A, Hu Y, Bax R, Page C (2002) The future challenges facing the development of new antimicrobial drugs. Nat Rev Drug Discov1: 895-910.

3. Brady SF (2007) Construction of soil environmental DNA cosmid libraries and screening for clones that produce biologically active small molecules. Nat protoc 2: 1297-1305

4. Li X, Qin L (2005) Metagenomics-based drug discovery and marine microbia diversity. Trends Biotechnol 23: 539-543.

5. Banik JJ, Brady SF (2010) Recent application of metagenomic approaches toward the discovery of antimicrobials and other bioactive small molecules. Curr Opin Microbiol 13: 603-609.

6. Daniel R (2005) The metagenomics of soil. Nat Rev Microbiol 3: 470-478.

7. Singh J, Behal A, Singla N, Joshi A, Birbian N, et al. (2009) Metagenomics: Concept, methodology, ecological inference and recent advances. Biotechno J 4: 480-494.

8. Kim JH, Feng Z, Bauer JD, Kallifidas D, Calle PY, et al. (2010) Cloning large natural product gene clusters from the environment: piecing environmental DNA gene clusters back together with TAR. Biopolymers 93: 833-844.

9. Feng Z, Kim JH, Brady SF (2010) Fluostatins produced by the heterologous expression of a TAR reassembled environmental DNA derived type II PKS gene cluster. J Am Chem Soc 132: 11902-11903

10. Craig JW, Chang FY, Brady SF (2009) Natural products from environmental DNA hosted in Ralstonia metallidurans. ACS Chem Biol 4: 23-28.
*Corresponding author: Zhi-Qiang Xiong, Key Laboratory of Synthetic Biology, Institute of Plant Physiology and Ecology, Shanghai Institutes for Biological Sciences, Chinese Academy of Sciences, 300 Fenglin Road, Shanghai 200032 China, Tel: 86-21-5492-4292; E-mail: zhqxiong@sibs.ac.cn

Received January 28, 2013; Accepted February 28, 2013; Published March 04 2013

Citation: Xiong ZQ (2013) Metagenomic-Guided Antibiotics Discovery. Clin Microbial 2: 101. doi:10.4172/2327-5073.1000101

Copyright: ( 2013 Xiong ZQ. This is an open-access article distributed under the terms of the Creative Commons Attribution License, which permits unrestricted use, distribution, and reproduction in any medium, provided the original author and source are credited. 